\title{
Patients undergoing dialysis as a model for medical students' longitudinal engagement with chronic illness: Patient perspectives
}

\author{
N. Koehler 1,2,3, E. L. Schmidt 1,2, M. A. Roberts ${ }^{1,4}$ \& J. Martin 1, 2,3,4
}

\begin{abstract}
Background: Medical students are predominantly exposed to patients with chronic conditions during acute episodes requiring hospitalisation. Consequently, this limits students' opportunities to learn about continuity of patient care. Unlike hospitalised patients, patients undergoing haemodialysis attend dialysis clinics in ambulatory/ outpatient settings multiple times per week over long time periods. Patients undergoing dialysis would be well placed to share their knowledge of their own chronic illness and their experience of patient-centred care. This study explored the willingness of patients undergoing haemodialysis to interact with the same medical student throughout the academic year.
\end{abstract}

Methods: Twenty-seven patients undergoing dialysis at four dialysis units were interviewed.

Results: Twenty-five patients indicated that they would be willing to regularly interact with students. Patients indicated that they would permit students to conduct a range of activities (e.g., taking a medical history). All 25 were willing to provide students with verbal feedback. Twelve were willing to provide written feedback. In terms of interacting with students, there were two predominant groups: 1) those who focus on the students' needs and are prepared to interact with students frequently and over a long duration and 2) those who have had "enough" after a while and are prepared to interact with students over a shorter duration.

\footnotetext{
Eastern Health Clinical School, Monash University, Victoria

Deakin University, Victoria

Monash Centre for Scholarship in Health Education, Monash University, Victoria

4 Eastern Health, Melbourne, Victoria
}

\section{Correspondence}

Associate Professor Jenepher Martin

Medical Education Research

Monash University Eastern Health Clinical School

Level 2, 5 Arnold St

Box Hill, VIC 3128

Australia

Tel: +61 402035631

Email: jenepher.martin@monash.edu 
Conclusions: Patients undergoing haemodialysis could provide students with opportunities to follow a patient's journey, provided the length and frequency of the patient-student interaction is matched with patient preferences.

Keywords: medical education; continuity of patient care; patient-centred care; chronic illness; longitudinal student/patient engagement; haemodialysis

\section{Introduction}

Medical students' clinical learning is predominantly in acute hospital settings. Shorter hospital stays, sicker patients and patients more aware of their rights to refuse students mean patient-student interactions have become limited (Braunack-Mayer, 2001; Wearn $\&$ Vnuk, 2005). In acute settings, students are only exposed to patients with chronic conditions during their acute admission, and these settings are not conducive to students following a patient longitudinally. Consequently, students' learning about the patient journey for management of chronic illnesses is limited. There is, thus, a need to explore opportunities outside the acute hospital setting for regular engagement with chronically ill patients.

Several medical schools have implemented longitudinal integrated rotation models emphasising the continuity of relationships between individual medical students and patients (Hudson et al., 2012; Norris et al., 2009; Poncelet \& Hudson, 2015). Patients value having multiple interactions with students and believe that these interactions help students improve important skills (e.g., communication with patients, understanding patients' perspectives and building rapport), enabling students to become more patient centred (Poncelet, Wamsley, et al., 2013). Students in a longitudinal integrated rotation reported having higher patient-centred attitudes at the end of their rotation compared to the beginning of their rotation, and the opposite occurred for students in a traditional program (Hirsh et al., 2012). A follow-up study showed that students' levels of patient centredness at the end of their rotation persisted 4-6 years post rotation, suggesting that the benefits of a longitudinal integrated rotation are sustained (Gaufberg et al., 2014).

Continuity with individual patients also helped students to understand a patient's illness from a physical and psychological perspective (Ogur \& Hirsh, 2009; Wamsley et al., 2009), understand how social factors impact on health (Ogur \& Hirsh, 2009), develop deeper connection and empathy with patients (Ogur \& Hirsh, 2009), improve their communication skills (Wamsley et al., 2009), improve understanding of chronic illness management (Ogrinc et al., 2002; Wamsley et al., 2009) and develop an understanding of the strengths and limitations of the healthcare system (Ogur \& Hirsh, 2009). Furthermore, students in a program with continuity of patients performed better on some examinations than those in a traditional program (Poncelet, Bokser, et al., 2011; Teherani et al., 2013).

Unlike hospital patients, patients with end-stage kidney disease (ESKD) who are receiving haemodialysis have regular contact with health services in an ambulatory setting over a long period. In Australia in 2018, of the 13,399 people with ESKD receiving any 
form of dialysis, there were 2,910 receiving dialysis in acute hospital settings and 7,016 in satellite facilities separate to an acute hospital, with the rest using a home-based therapy (ANZDATA, 2019). Comorbid conditions such as diabetes and coronary artery disease are present in about half of the patients receiving haemodialysis in a hospital or satellite setting (ANZDATA, 2019), and symptoms such as poor mobility, poor appetite, pruritus and depression are common (Davison et al., 2015). People receiving haemodialysis in a satellite facility usually regularly attend three 4-5 hour sessions a week. These patients may be suitable for individual students embedded in acute hospital contexts to regularly engage with the same patient in the same setting throughout the academic year. This model could assist students to develop skills associated with practising patient-centred care, deepen their understanding of chronic illness and appreciate the patient's journey over time.

The aim of this study was to explore the willingness of patients receiving regular ongoing treatment in an ambulatory care setting to interact with medical students during their treatment. Understanding this could inform the design of learning opportunities for students' sustained engagement with patients living with chronic illness.

\section{Method}

A mixed methods approach using individually administered questionnaire-based interviews explored patient perspectives about longitudinal interaction with medical students. Ethics approval was obtained from the Eastern Health Human Research Ethics Committee (LR20/2016).

\section{Context}

The setting for this study was a large, multi-site health service in metropolitan Melbourne, Australia. Four haemodialysis units are geographically distributed across the health service, co-located with acute care facilities, ranging in size from six to 20 chairs and providing haemodialysis to between 24 and 80 patients per week.

\section{Participants}

English-speaking patients aged over 18 years and receiving haemodialysis at one of four haemodialysis facilities were approached for interviews regarding their perceptions of engaging with medical students for educational purposes. No incentives or reimbursement was offered to participants.

\section{Interview format and administration}

A semi-structured, branched interview proforma was developed. Initial questions documented demographic and relevant clinical information. The remainder of the interview focused on exploration of possible student activities and discussion topics; frequency, length and longitudinal duration of patient-student interactions; and any concerns patients had about interacting with students (Appendix 1). No pilot was 
conducted, however the researchers met with the interviewer after two interviews were conducted to ascertain if amendments needed to be made. No amendments were required.

Interviews were conducted from August to November 2016 by a nurse experienced in conducting interviews with patients. Patients at each site identified by the nurse unit manager as having adequate English language and being well enough for interview were approached during a haemodialysis session. After written consent for participation was obtained, the structured questionnaire-based interview was conducted. The same interviewer conducted all interviews, with durations ranging from 30 to 40 minutes.

After documenting demographic information, participants were asked whether they would be prepared to see the same medical student for 30-60 minutes every 4 weeks whilst receiving dialysis throughout the year.

Those not prepared to see a medical student were asked about their related concerns. Follow-up questions explored specific potential factors of concern or influence for these participants, including what factors could change their perspective about seeing medical students.

Participants who were prepared to see a medical student were asked questions about their willingness to and comfort with permitting students to carry out various activities. These questions required an initial binary or 5-point scale response, followed by probing to explore reasons for that response. Subsequently, participants were asked questions relating to parameters for longitudinal student engagement, concerns about seeing students and what they thought were the most important things for students to learn.

\section{Data analysis}

Descriptive statistics and comparisons for quantitative data were analysed using the Statistical Package for the Social Sciences (SPSS) version 23. Thematic analysis of qualitative data relating to the questions about interaction length, frequency and duration was undertaken, with initial coding completed independently by two authors (NK, JM). Differences in categories and codes were resolved by consensus. After identification of principal themes, a second order analysis examined in-subject relationships between these themes.

\section{Results}

Of 36 patients approached, 27 (11 females) consented to participate (Site \#1: $\mathrm{n}=8$; Site $\# 2: \mathrm{n}=8$; Site \#3: $\mathrm{n}=6$; Site \#4: $\mathrm{n}=5)$, with a mean age of 69 years $(S D=11.9$; range = $25-82)$ and a mean of 4.5 years on haemodialysis $(S D=4.7$; range $=0.3-21)$. One patient had a previous kidney transplant. At the time of the interview, six patients were on the transplant waiting list and one was unsure about their waiting list status. 


\section{Willingness to see students}

Twenty-five patients (93\%) indicated they were willing to interact with a medical student regularly. Of the two who indicated that they would not be willing to do this, one explained she did not wish to be seen by students because she was too tired and her English was not very good. This patient declined to answer further questions. The other patient explained that she has a rare medical condition and already sees many medical students. As a vulnerable person, she expressed concern that students may attend the dialysis unit with colds or other infections. Whilst responding with "no" to the question about whether she had had bad experiences with students in the past, she indicated that the students themselves are not rude, but senior doctors assume she is willing to see students. The results that follow relate to the 25 patients participating in the full interview.

\section{Activities and discussion topics}

Of the remaining 25 participants, all would permit students to undertake the various activities and discuss relevant aspects of their ESKD, and they were generally comfortable with all activities and discussion topics (Table 1). Some patients indicated that the topics of paired kidney exchange $(n=9)$ and kidney transplant $(n=4)$ were "not applicable" to them, although only one indicated a lack of knowledge about transplants.

\section{Table 1}

Patients' Permissiveness and Level of Comfort With Various Activities Conducted by a Medical Student

\begin{tabular}{|c|c|c|c|c|c|c|c|c|}
\hline \multirow{2}{*}{ Activities and Discussion Topics } & \multicolumn{3}{|c|}{ Permit } & \multicolumn{5}{|c|}{ Level of Comfort } \\
\hline & Yes & No & N/A & 1 & 2 & 3 & 4 & 5 \\
\hline Take a medical history & 25 & & & & & & 8 & 17 \\
\hline Non-invasive physical examinations & 25 & & & & & 2 & 5 & 18 \\
\hline Current management & 25 & & & & & & 9 & 16 \\
\hline Personal perspective of dialysis & 25 & & & & & 1 & 7 & 17 \\
\hline Assist with clinical tasks under supervision & 25 & & & & & & 8 & 17 \\
\hline Dialysis in general & 25 & & & & & & 6 & 19 \\
\hline Fistulas & 25 & & & & & & 7 & 18 \\
\hline Kidney transplants & 21 & & 4 & & & 1 & 6 & 13 \\
\hline Paired kidney exchange program & 16 & & 9 & & & 1 & 3 & 12 \\
\hline Medications & 25 & & & & & & 6 & 19 \\
\hline Diagnosis/need for dialysis & 25 & & & & & 1 & 5 & 19 \\
\hline Other health concerns (not directly related to kidney function) & 25 & & & & & & 9 & 16 \\
\hline Interactions with health professionals during dialysis sessions & 25 & & & & & & 9 & 16 \\
\hline Interactions with health professionals outside of dialysis & 25 & & & & & 1 & 6 & 18 \\
\hline Living with a chronic illness & 25 & & & & & & 7 & 18 \\
\hline
\end{tabular}

Note: 1 = not comfortable; 5 = very comfortable. One participant did not provide a comfort rating for the "kidney transplants" topic. 


\section{Sessions}

All participants were prepared to see the same medical student for repeat visits, however they had varied opinions about how long an individual session should last. Approximately half suggested an interaction length of $\geq 60$ minutes, with four not giving a fixed time and stating they are happy to spend as much time as the student needed. In addition to a preference for session length, there were variations in frequency of interaction ( $\leq$ once a fortnight versus $\geq$ once a week) and duration of longitudinal engagement ( $\leq 6$ months versus $>6$ months) (Figure 1 ).

\section{Figure 1}

Patients' Preferences for Medical Student Sessions by Interaction Length, Interaction Frequency and Longitudinal Engagement

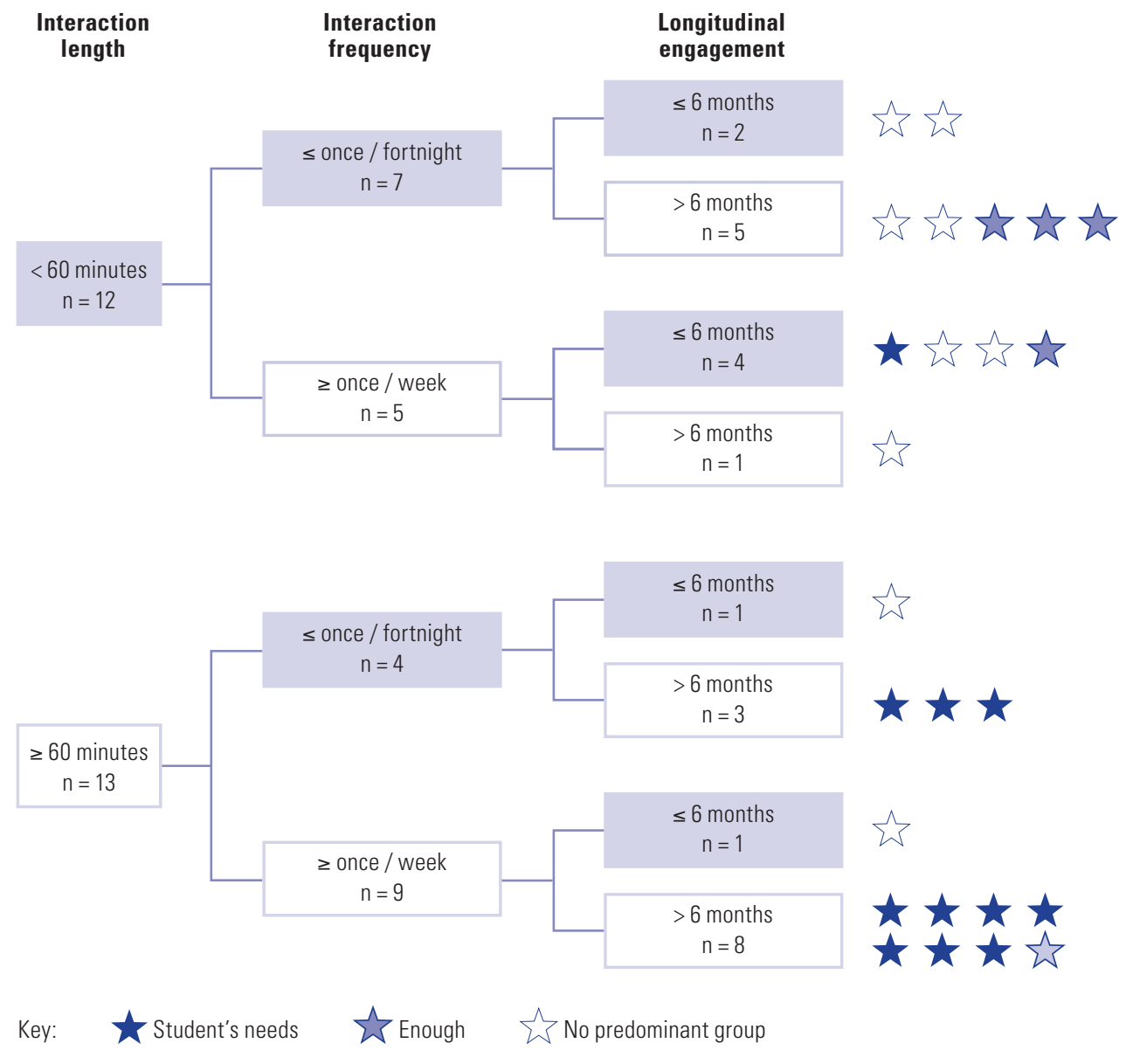

Note: Stars signify participant viewpoint based on thematic analysis of comments. 
Exploring each participant's preferences for sessions with medical students, we identified six overarching themes. Given the exploratory nature of this study, a theme was created if at least two patients made a related comment, so as to attach value to all patient perspectives that emerged and acknowledge that the questions were not framed to elicit a "most important" perspective. An exemplar of each theme is provided.

1) Meeting student needs: "The session should help the student learn. I'm happy for it to be as long as the student needs" (Patient 11). Patients identified their role and responsibility in helping students to learn. They recognised that students have a lot to learn and were prepared to accommodate student needs with respect to their time commitment.

2) Passing the time: "I'm here 5 hours so it would help pass the time" (Patient 19). For a number of patients, the opportunity to interact with a student would help pass the time during dialysis.

3) Enough time for the patient: "More than that could be too much, I might feel 'off' or tired" (Patient 3). In identifying their reasons for designating the ideal session interaction length, interaction frequency and longitudinal engagement, some patients clearly identified what was "enough" for them, commonly citing the potential for becoming tired.

4) Student sharing the patient's journey: "If I was successful at getting a transplant, the student could go through the journey with me" (Patient 14). Patients identified benefits to establishing a personal connection with individual students over time.

5) Specific reasons to limit interaction(s): "I have my dialysis days planned out. I like to read all the newspapers that I save for these days, and I like to listen to music" (Patient 6). Patients identified specific individual reasons for limiting the interaction length, interaction frequency and longitudinal engagement. For some patients, their own agenda for the day was important, and they did not want this disrupted.

6) Patient agency to determine frequency and longitudinal duration for student interactions: "Because that's all I want to do" (Patient 1). Patients expressed they wanted the flexibility to change or limit interactions if they found the commitment too onerous. In considering their longitudinal engagement with individual students, patients identified that students seeing a number of different patients may be beneficial.

Of interest, when within-subject themes were considered in regard to preferred session length, there were two predominant groupings. One $(n=11)$ was focused on meeting student needs, while the other $(\mathrm{n}=9)$ spoke about what was "enough" for them. Five participants did not clearly have one of these dominant perspectives. When we mapped these two themes onto the interaction length, frequency and longitudinal engagement preferences, 10 of the 11 participants in the "student needs" group indicated an interaction length $\geq 60$ minutes, with seven of these participants indicating an interaction frequency of $\geq$ once per week and a longitudinal duration of $>6$ months. Notably, seven 
out of the nine participants in the "enough" group indicated an interaction length of $<60$ minutes (Figure 1).

\section{Feedback}

All participants were prepared to provide students with verbal feedback on their interactions with them. Twenty-three participants (92\%) were prepared to do this after every session, whereas one participant preferred after every second session, and another preferred every few weeks. Comfort with providing verbal feedback was high $(M=4.6$; $S D=0.6$; range $=3-5)$. When it came to written feedback, $12(48 \%)$ participants would be prepared to provide this either at every session $(n=10)$, as often as the student wanted it $(n=1)$ or after every second session $(n=1)$. Thirteen patients were not prepared to provide written feedback, citing a number of reasons, including medical issues making writing difficult (e.g., poor eyesight, arthritis) ( $\mathrm{n}=4)$, not being good at writing/spelling $(\mathrm{n}=4)$, too difficult to write with one arm immobilised/lying down $(\mathrm{n}=3)$, the task being too onerous $(n=2)$ and English not being their first language $(n=1)$. For all participants, comfort levels with providing written feedback were significantly less than for verbal feedback $(M=2.8 ; S D=1.9$; range $=1-5 ; t 24=5.07, p \leq .001$ (two-tailed)).

\section{Most important things for students to learn}

Patients' responses regarding what they believed to be the most important things for students to learn fell into two categories:

1) Knowledge/technical aspects of kidney disease and dialysis $(\mathrm{n}=6)$, "the technical part (of dialysis) ..." (Patient 2).

2) Generic patient-centred consultation skills $(\mathrm{n}=18)$, "how to treat patients, how to listen to their concerns, have some empathy to feel the patient's pain" (Patient 14).

Some patients mentioned elements of both categories $(n=3)$, whereas others were not sure what they perceived to be important for students to learn $(n=2)$ or simply indicated "everything" ( $\mathrm{n}=4)$.

\section{Table 2}

Indication of Patients' Concern Regarding Interacting With Medical Students Under Various Conditions

\begin{tabular}{l|c|c}
\multicolumn{1}{c|}{ Concern } & Yes & No \\
\hline Students having colds or infectious conditions & 11 & 14 \\
\hline No supervisor sitting next to students & 0 & 25 \\
\hline Students' levels of knowledge & 0 & 25 \\
\hline Students interfering with treatment & 2 & 23 \\
\hline Being too tired to see students & 2 & 23 \\
\hline Being too unwell to see students & 2 & 23 \\
\hline
\end{tabular}




\section{Concerns}

The most frequent concern indicated by patients was that students may come to the dialysis unit with a cold or another infectious condition (Table 2).

Our results suggest that people receiving haemodialysis fall into three groups with respect to seeing medical students over the course of a year: 1) those who do not wish to interact with students, 2) those who are willing to interact with students over a brief period of time and 3) those who are willing to interact with students over a lengthy period of time. For most student activities and discussion topics, a high level of comfort was demonstrated.

\section{Discussion}

Achieving longitudinal engagement of medical students with patients is challenging (Mol et al., 2019), and this study suggests that a "one-size-fits-all" model is not appropriate for patients. Better understanding patients' perspectives reveals opportunities for co-creation with patients of longitudinal student/patient engagement models and potential benefits to students of "lived experience" delivery of curriculum content and development of skills for patient-centred care.

Providing opportunities for patients to participate in the design and delivery of health professional education is one of the key requirements for patient-centred care (Ogden et al., 2017). Cribb et al. (2017) identify that co-creation with patients in medical education is an opportunity for medical schools to role model underpinning principles of patientcentred care and contribute to transformative change in healthcare. In keeping with this is the meta-narrative of "democratization of health professions education" identified by Rowland et al. (2019), whereby "expert and engaged" patients are involved in medical education. In our context, patients undergoing haemodialysis have a high level of comfort with students undertaking clinical activities and discussing topics related to the patient's medical condition with them, identifying both knowledge of kidney disease/dialysis and patient-centred consultation skills as the most important aspects of student learning. These patients are willing to actively engage as educators, and there is potential to work collaboratively with them to design curriculum delivery for development of both medical knowledge and patient-centred skills.

Those patients willing to engage with students see themselves as having an active education role, and our study highlights the importance of identifying those who are willing and then working within their preferences. Planning for student placements would ideally explore individual patient preferences for session frequency, length and longitudinal engagement. However, considering that there were two identifiable groups of preferences for student interaction, a template approach for high or low student interaction could be more feasible. This approach could also identify patients for single intermittent sessions rather than continuous interactions over longer periods of time. 
Further research is required to better understand differences in motivation to participate and perceptions of the educational role for the two groups.

Previous research shows that there are various reasons patients volunteer to interact with medical students. These reasons are not necessarily mutually exclusive and include giving back to the medical profession, out of gratitude for someone having saved their life, that they or a family member studied at the same university as the medical students or because someone recommended that they participate (Koehler \& McMenamin, 2013). Another important consideration is the active management of patient-perceived concerns about students in the clinical setting. Patients undergoing haemodialysis were not concerned about students interfering with their treatment, however they had concerns about infection risks, and mitigating these risks through the use of strategies such as hand hygiene, exclusion of unwell students and student vaccination are important considerations for program design.

Benefits for student learning through longitudinal patient-student engagement include increased patient-centred attitudes (Gaufberg et al., 2014; Hirsh et al., 2012; Poncelet, Wamsley, et al., 2013) and development of professional relationships and communication (Wamsley et al., 2009) over the course of a patient journey. These benefits to students were recognised by our patient participants highlighting that patients, like medical educators, perceive learning patient-centred skills to be of high importance.

Furthermore, some patients directly commented on sharing their journey with a student, indicating the importance of students obtaining a longitudinal perspective of care. Each patient's journey is unique and unpredictable. For example, patients may change their treatment modality (e.g., changing from haemodialysis in a satellite clinic to home dialysis), have hospital admissions related to their renal failure or other medical issues or have significant personal factors affecting their life. Following patients longitudinally enables students to learn how these factors interplay with the patients' health and wellbeing and how patients navigate these challenges and changes in their lives. In addition to gaining medical knowledge, students learn to see the patient with an illness instead of focusing on the disease process and content knowledge. Additionally, longitudinal interaction with a patient provides opportunities for development of communication skills and empathy.

Longitudinal engagement between one patient and one student provides an opportunity for patients to provide specific feedback to students rather than feedback on a generic set of skills. This feedback can be in verbal and/or written form. Our study indicates that patients are more comfortable with providing students with verbal feedback, citing medical issues and poor literacy skills as reasons for not wishing to provide written feedback. This suggests that educators may need to assist individual patients in providing written feedback if considered desirable. 
When developing curricula for longitudinal patient-student engagement, it is important to make the explicit objectives transparent to students, patients and educators. Students may not understand how such interactions can help with their acquisition of clinical and social knowledge and patient-centred skills without this transparency of purpose. Patients' lived experience of chronic illness can provide students with a depth of understanding from an expert perspective not usually covered in a textbook or even through simulation (Towle \& Godolphin, 2011), and patients may need specific orientation to the value of this prior to participation.

\section{Limitations}

This study is exploratory, and in a single context (outpatient haemodialysis), so the transferability of the findings to other settings in acute health services (e.g., day oncology, mental health services) that may provide opportunities for longitudinal student-patient engagement cannot be assumed. Additionally, the findings may not be applicable in other dialysis settings (e.g., inpatient or home). Furthermore, it must be noted that responses to questions were short and focused, limiting the richness of the data. The findings do, however, provide the basis for exploration of other contexts to build a model for curriculum design of health professional students' longitudinal engagement with patients.

We recognise that the patients interviewed were a selected group, first, identified as having English language skills and being well enough for interview and, second, by agreeing to interview. With only two participants unwilling to see students, we have very limited understanding of reasons for this. It is possible a higher proportion of patients who declined to participate would not be willing to see students. A pragmatic perspective, however, is that screening of patients will be necessary in developing successful longitudinal student-patient engagement. Educators also need to recognise that patients may decline to interact with students due to unforeseen reasons even after the engagement has commenced.

\section{Conclusion}

People receiving haemodialysis in an ambulatory setting could provide students with the opportunity to follow a patient's journey through different stages of their condition. In this study, interviewees demonstrated a high level of comfort in talking about their condition, with many prepared to have regular interactions with medical students over time. Exposure of medical students to such people outside the acute hospital environment would assist the student in developing empathy and understanding of what it is like to live with a chronic illness, which in turn could improve patient-centred care by future clinicians.

\section{Acknowledgements}

NK thanks her late friend and mentor, Prof Christine McMenamin, for her inspiration and thought-provoking discussion regarding this study whilst undergoing renal dialysis. 
We thank the dialysis nurse unit managers for permission to approach patients, Margaret Curtis for conducting the interviews and patients and dialysis unit staff who assisted with our study. Aspects of this study were presented at the Australian \& New Zealand Association for Health Professional Educators (ANZAHPE) conference, Adelaide, Australia, 2017 and the International Clinical Skills Conference, Prato, Italy, 2019.

\section{Conflicts of interest and funding}

NK acknowledges that interview questions related to activities and discussion topics were based on her observations of her late friend and mentor whilst undergoing haemodialysis on a weekly basis over a 12-month period. NK's late friend and mentor was not a participant in the study nor a patient at the haemodialysis units within this study. Questions pertaining to patients' concerns were based on feedback the authors commonly received from patients in regards to interacting with students at their clinical school. Funding support was from the Monash Education Academy Small Grants Scheme 2015, Monash University.

\section{References}

ANZDATA: Australia \& New Zealand Dialysis \& Transplant Registry. (2019). Chapter 2: Prevalence of renal replacement therapy for end stage kidney disease. In ANZDATA 42nd annual report 2019 (Date to 2018). https://www.anzdata.org.au/wpcontent/uploads/2019/09/c02_prevalence_2018_ar_2019_v1.0_20191202.pdf

Braunack-Mayer, A. J. (2001). Should medical students act as surrogate patients for each other? Medical Education, 35(7), 681-686. https://doi.org/10.1046/j.13652923.2001.00970.x

Cribb, A., Owens, J., \& Singh, G. (2017). Co-creating an expansive health care learning system. American Medical Association Journal of Ethics, 19(11), 1099-1105. https://doi. org/10.1001/journalofethics.2017.19.11.medu1-1711

Davison, S. N., Levin, A., Moss, A. H., Jha, V., Brown, E. A., Brennan, F., Murtagh, F. E. M., Naicker, S., Germain, M. J., O’Donoghue, D. J., Morton, R. L., \& Obrador, G. T. (2015). Executive summary of the KDIGO controversies conference on supportive care in chronic kidney disease: Developing a roadmap to improving quality care. Kidney International, 88(3), 447-459. https://doi.org/10.1038/ $\underline{\text { ki.2015.110 }}$

Gaufberg, E., Hirsh, D., Krupat, E., Ogur, B., Pelletier, S., Reiff, D., \& Bor, D. (2014). Into the future: Patient-centredness endures in longitudinal integrated clerkship graduates. Medical Education, 48(6), 572-582. https://doi.org/10.1111/medu.12413 
Hirsh, D., Gaufberg, E., Ogur, B., Cohen, P., Krupat, E., Cox, M., Pelletier, S., \& Bor, D. (2012). Educational outcomes of the Harvard Medical School-Cambridge integrated clerkship: A way forward for medical education. Academic Medicine, 87(5), 643-650. https://doi.org/10.1097/ACM.0b013e31824d9821

Hudson, J. N., Knight, P. J., \& Weston, K. M. (2012). Patient perceptions of innovative longitudinal integrated clerkships based in regional, rural and remote primary care: A qualitative study. BMC Family Practice, 13, Article 72. https://doi.org/10.1186/14712296-13-72

Koehler, N., \& McMenamin, C. (2013). Patients' attitudes towards participating in clinical skills training purely for teaching purposes. International Journal of Clinical Skills, 7(2), 23-27. https://www.ijocs.org/clinical-journal/patients-attitudes-towardsparticipating-in-clinical-skills-training-purely-for-teaching-purposes.pdf

Mol, S. S. L., Chen, H. C., Steerneman, A. H. M., de Groot, E., \& Zwart, D. L. M. (2019). The feasibility of longitudinal patient contacts in a large medical school. Teaching and Learning in Medicine, 31(2), 178-185. https://doi.org/10.1080/10401334. $\underline{2018.1524330}$

Norris, T. E., Schaad, D. C., DeWitt, D., Ogur, B., Hunt, D. D., \& Consortium of Integrated Longitudinal Clerkships. (2009). Longitudinal integrated clerkships for medical students: An innovation adopted by medical schools in Australia, Canada, South Africa, and the United States. Academic Medicine, 84(7), 902-907. https://doi. org/10.1097/ACM.0b013e3181a85776

Ogden, K., Barr, J., \& Greenfield, D. (2017). Determining requirements for patientcentred care: A participatory concept mapping study. BMC Health Services Research, 17(1), Article 780. https://doi.org/10.1186/s12913-017-2741-y

Ogrinc, G., Mutha, S., \& Irby, D. (2002). Evidence of longitudinal ambulatory care rotations: A review of the literature. Academic Medicine, 77(7), 688-693. https://doi. org/10.1097/00001888-200207000-00008

Ogur, B., \& Hirsh, D. (2009). Learning through longitudinal patient care: Narratives from the Harvard Medical School-Cambridge integrated clerkship. Academic Medicine, 84(7), 844-850. https://doi.org/10.1097/ACM.0b013e3181a85793 
Poncelet, A., Bokser, S., Calton, B., Hauer, K. E., Kirsch, H., Jones, T., Lai, C. J., Mazotti, L., Shore, W., Teherani, A., Tong, L., Wamsley, M., \& Robertson, P. (2011). Development of a longitudinal integrated clerkship at an academic medical center. Medical Education Online, 16(1), Article 5939. https://doi.org/10.3402/meo. $\underline{\mathrm{v} 16 \mathrm{i} 0.5939}$

Poncelet, A. N., Wamsley, M., Hauer, K. E., Lai, C., Becker, T., \& O’Brien, B. (2013). Patient views of continuity relationships with medical students. Medical Teacher, 35(6), 465-471. https://doi.org/10.3109/0142159X.2013.774335

Poncelet, A. N., \& Hudson, J. N. (2015). Student continuity with patients: A system delivery innovation to benefit patient care and learning (continuity patient benefit). Healthcare, 3(3), 607-618. https://doi.org/10.3390/healthcare3030607

Rowland, P., Anderson, M., Kumagai, A. K., McMillan, S., Sandhu, V. K., \& Langlois, S. (2019). Patient involvement in health professionals' education: A meta-narrative review. Advances in Health Sciences Education Theory and Practice, 24(3), 595-617. https://doi.org/10.1007/s10459-018-9857-7

Teherani, A., Irby, D. M., \& Loeser, H. (2013). Outcomes of different clerkship models: Longitudinal integrated, hybrid, and block. Academic Medicine, 88(1), 35-43. https:// doi.org/10.1097/ACM.0b013e318276ca9b

Towle, A., \& Godolphin, W. (2011). A meeting of experts: The emerging roles of nonprofessionals in the education of health professionals. Teaching in Higher Education, 16(5), 495-504. https://doi.org/10.1080/13562517.2011.570442

Wamsley, M. A., Dubowitz, N., Kohli, P., Cooke, M., \& O’Brien, B. C. (2009). Continuity in longitudinal out-patient attachment for year 3 medical students. Medical Education, 43(9), 895-906. https://doi.org/10.1111/j.13652923.2009.03424.x

Wearn, A., \& Vnuk, A. (2005). Medical students and peer physical examination: Two case studies of strategies to improve safety and increase acceptance. Focus on Health Professional Education: A Multi-Professional Journal, 7(2), 88-98. https://dspace. flinders.edu.au/xmlui/handle/2328/12234 


\section{Appendix 1}

Interview Questions and Questioning Route

\section{Summary of interview questions}

\section{Background information}
Dialysis unit: $\square$ site $1 \square$ site $2 \square$ site $3 \square$ site 4
Gender: $\quad \square$ female $\square$ male $\square$ other
Age: ___ years

How long have you been coming to a dialysis unit? (weeks / months / years) or date (e.g., since mid-April 2014)

Have you had a kidney transplant previously?

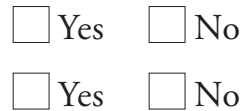

Are you on a waiting list for a kidney transplant? $\quad \square$ Yes $\square$ No

\section{Interacting with students}

Prompt question: Would you be prepared for a medical student to see you whilst you are undergoing dialysis for 30-60 mins every 4 weeks throughout the year? $\square$ Yes $\square$ No

"No" response to prompt: Could you please explain what your concerns or fears might be in regards to seeing a medical student?

Follow up: Thank you for your explanation as to why you do not wish to see students. Do any of the following contribute to your response for not wishing to see students?

- Concerned that students may come into the dialysis unit with colds or other infectious conditions

- Concerned that students will not have a supervisor sitting next to them whilst they engage with you

- Concerned about students' levels of knowledge given their year level

- Concerned that students may interfere with the treatment that you are receiving

- Being too tired to see students

- Being too unwell to see students

- Having seen many students/interns/residents in the past and you would like a break

- Bad experiences with students previously

Is there anything that could be done in order for you to change your mind about students seeing you whilst you are undergoing dialysis? 
Is there anything that was missed during the interview that you feel is important in regards to medical students interacting with patients on dialysis?

\section{"Yes" response to prompt:}

1. Questions exploring permission to undertake, and comfort with, clinical activities (take a medical history, conduct non-invasive physical examinations, assist with clinical tasks under supervision, discuss current medical management, personal perspective about experience on dialysis) and more general discussion topics (dialysis in general, fistulas, kidney transplants, paired exchange program, medications, personal diagnosis/need for dialysis, their other health concerns not related to kidney function, interactions with health professionals during dialysis, interactions with health professionals outside dialysis sessions, living with chronic illness).

i. Would you permit a medical student to [insert activity/discussion topic here, e.g., take a medical history]?

ii. How comfortable are you with a medical student carrying out [the named activity] or discussing [discussion topic]? Comfort rated scale: 1 (not comfortable) 5 (very comfortable).

2. Questions exploring willingness and comfort with providing verbal and written feedback to students.

i. Are you prepared to provide students with (insert "verbal" or "written") feedback following their interaction with you?

If no: explore why

If yes: How often are you willing to provide students with (insert "verbal" or "written") feedback (e.g., after every session, retrospectively after x number of sessions)? Rate comfort (1-5),

Frequency of interactions (interviewer permitted to orient participant to term "session", e.g., minutes/hours; "frequency", e.g., per week/month; "length of time", e.g., weeks/months/year)

(i) Would you be prepared to see the same medical student multiple times (i.e., repeat visits)?

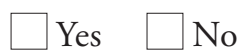

(ii) What do you perceive to be the ideal length of a session with a medical student?

Why do you think that [insert patient's response here] is ideal?

(iii) How frequently are you willing to interact with medical students?

Why do you think that [insert patient's response here] is ideal?

(iv) Over what length of time are you willing to interact with medical students? 
Why do you think that interacting with a student over [insert patient's response here] is ideal?

The interviewer to then follow up with exploration of each participant's suitable days/ times purely to provide an overview for future curriculum planning as to likely spread of opportunities for students.

\section{Concerns}

Prompt question: Do you have any concerns in regards to medical students interacting with patients on dialysis purely for educational purposes? $\square$ Yes $\square$ No

"Yes" response to prompt: Open question regarding the concerns: What are your concerns?

All respondents: Exploration of specific concerns: Would any of the following be of concern to you?

- Students may come into the dialysis unit with colds or other infectious conditions

- Students will not have a supervisor sitting next to them whilst they engage with you

- Students' levels of knowledge given their year level

- Students may interfere with the treatment that you are receiving

- Being too tired to see students

- Being too unwell to see students

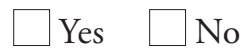

\section{General}

What do you believe is most important for students to learn from their interaction with patients on dialysis?

Is there anything that was missed during the interview that you feel is important in regards to medical students interacting with patients on dialysis? 